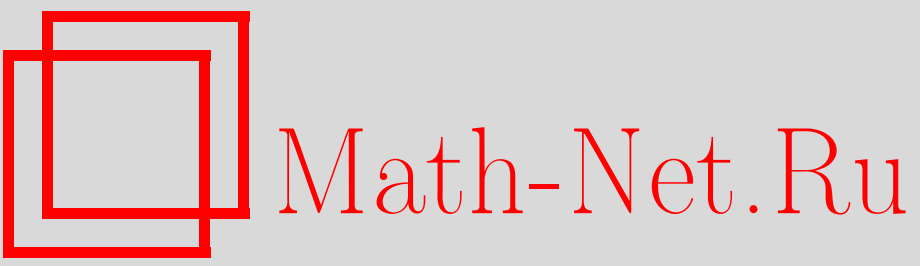

М. В. Зайцев, С. П. Мищенко, Асимптотика функций роста кодлины многообразий алгебр Ли, УМН, 1999, том 54, выпуск 3, 161-162

DOI: https://doi.org/10.4213/rm173

Использование Общероссийского математического портала Math-Net.Ru подразумевает, что вы прочитали и согласны с пользовательским соглашением

http://www.mathnet.ru/rus/agreement

Параметры загрузки:

IP: 54.174 .149 .18

26 апреля 2023 г., 17:06:22 


\title{
АСИМПТОТИКА ФУНКЦИЙ РОСТА КОДЛИНЫ МНОГООБРАЗИЙ АЛГЕБР ЛИ
}

\author{
М.В. ЗАЙцев, С.П. МищЕнкО
}

В работе изучаются числовые характеристики многообразий алгебр Ли, важнейшими из которых являются коразмерность тождеств и кодлина. Асимптотическое поведение коразмерности тождеств является важным классификационным признаком многообразия, существенно влияет на его свойства и изучалось в большом числе работ. Асимптотика функции кодлины для многообразий алгебр Ли, ее связь с другими числовыми характеристиками исследованы гораздо хуже. В данной работе показано, что существуют многообразия с экспоненциальным и сверхэкспоненциальным ростом кодлины и вычислена асимптотика кодлины для произведения двух нильпотентных многообразий. Все необъясняемые понятия можно найти в монографиии [1].

Характеристика основного поля $\Phi$ предполагается равной нулю. В лиевских элементах договоримся опускать скобки при их левонормированной расстановке, то есть $a b c=((a b) c)$.

Обозначим через $F=F(X, \mathbf{V})$ относительно свободную алгебру многообразия $\mathbf{V}$ со счетным множеством свободных образующих $X=\left\{x_{1}, x_{2}, \ldots\right\}$. Обозначим через $P_{n}=P_{n}(\mathbf{V})$ совокупность всех полилинейных элементов от $x_{1}, \ldots, x_{n}$ в пространстве $F$. Действие $\sigma\left(x_{i}\right)=x_{\sigma(i)}$ симметрической группы $S_{n}$ естественным образом продолжается на все пространство $P_{n}$. Исследование структуры $P_{n}$ как $S_{n}$-модуля играет важную роль при изучении многообразия $\mathbf{V}$.

Обозначим через $\chi_{\lambda}$ характер неприводимого представления симметрической группы, соответствуюшего разбиению $\lambda$ числа $n$, и рассмотрим для многообразия $\mathbf{V}$ разложение

$$
\chi_{n}(\mathbf{V})=\chi\left(P_{n}(\mathbf{V})\right)=\sum m_{\lambda} \chi_{\lambda} .
$$

Асимптотическое поведение размерности $c_{n}=c_{n}(\mathbf{V})$ пространства $P_{n}=P_{n}(\mathbf{V})$ определяет рост многообразия. Число слагаемых

$$
l_{n}=l_{n}(\mathbf{V})=\sum m_{\lambda}
$$

в сумме (1) называют кодлиной многообразия. Важными числовыми характеристиками многообразия являются также кратности $m_{\lambda}$ в сумме (1).

Обозначим через $d_{\lambda}$ размерность соответствующего $\lambda$ неприводимого модуля. Отметим, что для введенных числовых характеристик выполняется такое соотношение

$$
c_{n}(\mathbf{V})=\operatorname{dim} P_{n}(\mathbf{V})=\sum m_{\lambda} d_{\lambda}
$$

Напомним, что в случае многообразий ассоциативных алгебр рост кратностей и кодлина многообразия ограничены полиномиальной функцией $n^{q}$ для подходящего $q$ (см., например, [2; теорема 16]). Как было показано авторами, в случае алгебр Ли это свойство остается верным для так называемых многообразий ассоциативного типа и может нарушаться в общем случае (см. [3]). Однако в предложенных ранее примерах рост кратностей и кодлины оставался субэкспоненциальным.

В данной работе приводятся примеры многообразий, для которых кодлина является сверхэкспоненциальной функцией. Из асимптотической оценки числа различных разбиений числа $n$ следует, что в этом случае будут существовать и кратности с таким же ростом. Кроме того, в данной статье для любого натурального $b \geqslant 2$ приводится пример многообразия, кодлина которого ведет себя как функция $b^{n / 2}$.

Сформулируем основные результаты.

Работа выполнена при частичной поддержке Российского фонда фундаментальных исследований (гранты №№ 99-01-00233, 98-01-01020 и 96-15-96050.) 
Теорема 1. Кодлинъ многообразий $\mathbf{A}^{3}$ и $\mathbf{A N}_{3}$ растут сверхәкспоненциальным образом.

Теорема 2. Кодлина многообразия $\mathbf{N}_{b} \mathbf{N}_{2}, b \geqslant 2$, асимптотически совпадает с әкспоненциальной функцией $b^{n / 2}$, m.e. $l_{n}\left(\mathbf{N}_{b} \mathbf{N}_{2}\right) \simeq(\sqrt{b})^{n}$.

Приведем сначала ряд примеров многообразий со сверхэкспоненциальной кодлиной. Для построения нужных примеров нам потребуется ряд нижних оценок. Отметим сначала, что из формул (2), (3) и соотношения $n !=\sum_{\lambda \vdash n} d_{\lambda}^{2}$ легко вытекает следуюшее утверждение.

ПРЕДЛОЖЕНИЕ 1. Кодлина и коразмерность любого многообразия линейных алгебр связань неравенством: $l_{n}(\mathbf{V}) \geqslant \frac{c_{n}(\mathbf{V})}{\sqrt{n !}}$.

Приведем теперь примеры многообразий алгебр Ли, кодлина которых не может быть ограничена экспоненциальной функцией.

В работе [4] найдена асимптотика роста полинильпотентных многообразий. На основе этих асимптотик и предложения 1 получаем нижние оценки кодлин этих многообразий. Так, для многообразия $\mathbf{A}^{3}$ всех разрешимых ступени не выше трех алгебр Ли получаем

$$
c_{n}\left(\mathbf{A}^{3}\right) \simeq \frac{n !}{(\ln n)^{n}}, \quad l_{n}\left(\mathbf{A}^{3}\right) \geqslant \frac{\sqrt{n !}}{(\ln n)^{n}} .
$$

Многообразие $\mathbf{V}=\mathbf{N}_{s_{q}} \mathbf{N}_{s_{q-1}} \cdots \mathbf{N}_{s_{1}}$ при числе сомножителей $q \geqslant 3$ может иметь еще более значительную кодлину. Обозначим через $\ln ^{(k)} x k$-кратную композицию логарифома, т.е. $\ln ^{(k)} x=\ln (\ln (\ldots x) \ldots)$. Для такого многообразия в работе [4] получена оценка коразмерности

Tеорема ([4; теорема 2.2]). Пусть $\mathbf{V}=\mathbf{N}_{s_{q}} \mathbf{N}_{s_{q-1}} \cdots \mathbf{N}_{s_{1}}$. Тогда

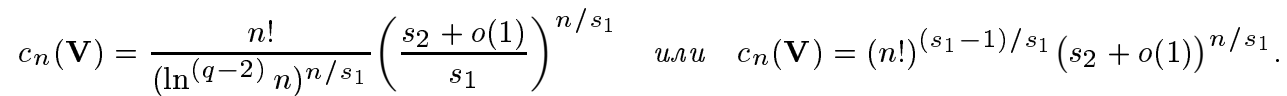

Первая из формул относится к случаю $q \geqslant 3$, а вторая получена для $q=2$. Отсюда следует

ПреДЛОЖениЕ 2. Для полинильпотентных многообразий алгебр Ли выполнены следующие соотношения

$$
l_{n}\left(\mathbf{N}_{s_{q}} \mathbf{N}_{s_{q-1}} \cdots \mathbf{N}_{s_{1}}\right) \geqslant \frac{\sqrt{n !}}{\left(\ln ^{(q-2)} n\right)^{n / s_{1}}} \quad(q \geqslant 3), \quad l_{n}\left(\mathbf{N}_{b} \mathbf{N}_{a}\right) \geqslant(\sqrt{b})^{n}(n !)^{(a-2) /(2 a)} .
$$

В частности, при $a \geqslant 3$ кодлина $\mathbf{N}_{b} \mathbf{N}_{a}$ растет сверхэкспоненциальным образом.

ЗАмЕчАниЕ. Существуют многообразия алгебр Ли со сверхэкспоненциалњными кратностями для некоторых неприводимых модулей.

Это следует из известной (см., например, [5]) асимптотики числа $p(n)$ различных разбиений $n$, $p(n) \sim \frac{1}{4 n \sqrt{3}} \exp \left(\pi \sqrt{\frac{2 n}{3}}\right)$. Отметим также, что в работе [6] получена следующая оценка кодлины многообразия $\mathbf{A N}_{2}: l_{n}\left(\mathbf{A N}_{2}\right) \sim \exp \left(\sqrt{\frac{2}{3} n \pi}\right)$. Для данного многообразия рост кодлины оказался промежуточным между полиномиальным и экспоненциальным.

\section{СПИСОК ЛИТЕРАТУРЫ}

[1] Бахтурин Ю. А. Тождества в алгебрах Ли. М.: Наука, 1985. [2] Berele A., Regev A. // J. Algebra. 1983. V. 82. P. 559-567. [3] Зайцев М. В., Мищенко С. П. О полиномиальности роста кодлины многообразий алгебр Ли // Алгебра и логика (в печати). [4] Петроградский В. М. // Матем. сб. 1997. Т. 188. №6. С. 119-138. [5] Эндрюс Г. Теория разбиений. М.: Наука, 1982. [6] Giambruno A., Mishchenko S., Zaicev M. On the colength of a variety of Lie algebras // Internat. J. Algebra Comput. (to appear). 